\title{
SOME NEW HILBERT ALGEBRAS
}

\author{
BY \\ R. $\operatorname{KEOWN}\left({ }^{1}\right)$
}

1. Introduction. The object of the present paper is the introduction of a new class of commutative Hilbert algebras. These algebras are distinct from those produced by a number of variations of the original axioms of an $H^{*}$-algebra given by W. Ambrose [1], [2]. The structure theorems obtained in the previous modifications give decompositions of the algebra into orthogonal subspaces, each of which is a minimal left ideal. In the commutative case, these result in an orthogonal basis for the space consisting of minimal, usually selfadjoint, idempotents. By adopting a different set of axioms, the present author determines a more general set of commutative $H$-algebras in which the direct sum decomposition is not necessarily an orthogonal one. The set $\left\{e_{i}\right\}$ of minimal idempotents which appear need neither be selfadjoint nor orthogonal, but rather make up the elements of a Fischer-Riesz system, a concept introduced by N. Bary [3]. A brief summary of the theory of Bary's is given in $\$ 4$.

This paper obtains structure theorems for three different formulations of the axioms. $\$ 2$ discusses the concept of a regular algebra in which the fundamental assumption is that every maximal modular ideal $R$ have a complementary ideal. $\S 3$ introduces the concepts of an adjoint algebra and of a dual adjoint algebra. An adjoint algebra is an algebra with two binary operations which permit the appearance of nonselfadjoint idempotents in the commutative case. A dual adjoint algebra is an algebra with two inner products which again permit the occurrence of nonselfadjoint idempotents. $\$ 4$ gives a brief summary of the concepts of Bessel system, Hilbert system, and Fischer-Riesz system introduced by N. Bary. It also contains a basic example of a more general type of $H$-algebra than the standard $H^{*}$-algebra.

The symbols $\left\{e_{i}\right\},\left\{g_{i}\right\},(i=1,2, \ldots)$, are used to denote the elements of a biorthogonal system in a Hilbert space $H$. The symbols $\left(e_{i}\right)$ and $\left[e_{i}\right]$ denote the linear hull and the closed linear hull respectively of the set of vectors $\left\{e_{i}\right\}$, $(i=1,2, \ldots)$. All of the algebras which appear in this paper are semisimple, commutative $H$-algebras, although this condition is not always expressly stated. The continuity in multiplication is given by the existence of a constant $K$ such that

$$
\|x y\| \leqq K\|x\|\|y\| \text {. }
$$

All of the Hilbert spaces which appear as underlying spaces of the algebras are assumed to be separable. This convention is used primarily for notational convenience.

Presented to the Society, September 1, 1966; received by the editors September 7, 1965.

(1) This research was supported by the FRIO Charitable Foundation, Houston, Texas. 
2. Complemented algebras. All of the Banach algebras considered in $\S 2$ are semisimple, commutative $H$-algebras. An algebra $A$ is regular if every maximal modular ideal $R$ of $A$ is complemented, i.e., there exists an ideal $I$ of $A$ such that $A$ is the direct sum $R+I$.

LEMMA 2.1. Every maximal modular ideal $R$ of a regular algebra $A$ has associated with it a unique minimal idempotent $e$ and a unique multiplicative element $g$. An element $x \in A$ is in $R$ if and only if either ex is the zero vector or $(x, g)$ is the zero complex number. For every pair $x, y \in A$,

$$
(x y, g)=(x, g)(y, g), \quad \text { and } \quad e x=(x, g) e .
$$

Proof. The difference algebra $A / R$ is isomorphic to the complex numbers so that the natural mapping $n$ of $A$ onto $A / R$ can be regarded as a linear functional on $A$. Since $R$ is closed, $n$ is a bounded linear functional. Thus there exists a unique element $g \in A$ so that $n(x)$ and $(x, g)$ are equal for every $x \in A$. Since $n$ is a homomorphism of $H$,

$$
(x y, g)=n(x y)=n(x) n(y)=(x, g)(y, g)
$$

for every $x, y \in A$. This unique $g$ is called the associated multiplicative element of $R$. Note that if $e$ is any idempotent, then

$$
(e, g)=\left(e^{2}, g\right)=(e, g)^{2}
$$

so that $(e, g)$ must be either one or zero.

There exists at least one ideal $I$ complementary to $R$. Since $I$ is isomorphic as a vector space to $A / R$, it must be a one-dimensional minimal ideal of $A$. The idempotent generator $e$ of $I$ is a minimal idempotent of $A$. If $I$ and $I^{\prime}$ are distinct complementary ideals of $R$ with idempotent generators $e$ and $e^{\prime}$ respectively, the product $e e^{\prime}$ must be zero. Consequently,

$$
0=\left(e e^{\prime}, g\right)=(e, g)\left(e^{\prime}, g\right) .
$$

It follows that either $e$ or $e^{\prime}$ must be contained in $R$, a contradiction. Therefore the complementary minimal ideal $I$ and the minimal idempotent generator $e$ are uniquely determined by the maximal modular ideal $R$.

Note that $e x$ must be a multiple $\pi e$ of $e$ for any $x \in A$. Since $(e, g)$ is one, we see that

$$
e x=(x, g) e .
$$

A slight variation of the above argument gives the

LEMMA 2.2. Every minimal idempotent e of a regular algebra $A$ is the idempotent associated with some maximal regular ideal $R$ of $A$.

We emphasize the useful fact that $e x$ is zero for a minimal idempotent $e$ if and only if $(x, g)$ is zero for the associated multiplicative element $g$, i.e., if and only if $x$ is an element belonging to the corresponding maximal regular ideal $R$. 
Lemma 2.3. Let $\left\{R_{\pi}\right\},\left\{e_{\pi}\right\},\left\{g_{\pi}\right\},(\pi \in P)$, denote the set of all maximal modular ideals, associated minimal idempotents, and associated multiplicative elements respectively of a regular algebra $A$. Then

$$
\begin{aligned}
\left(e_{\pi}, g_{\pi^{\prime}}\right) & =1, & \pi=\pi^{\prime}, \\
& =0, & \pi \neq \pi^{\prime} .
\end{aligned}
$$

This lemma is merely a restatement of the preceding two. Nevertheless, it points up the important fact that the two systems $\left\{g_{\pi}\right\}$ and $\left\{e_{\pi}\right\}$ form a biorthogonal system for a regular algebra $A$. It follows immediately from semisimplicity that the system $\left\{g_{\pi}\right\}$ is complete.

LEMMA 2.4. A regular algebra A contains at most a countably infinite number of distinct maximal regular ideals whenever $A$ is a separable Hilbert space.

Proof. The set $M$ of multiplicative elements $\left\{g_{\pi}\right\}$ is a part of a biorthogonal system. By a result of S. Levin [7], no element $g$ of $M$ is contained in the closed linear hull $\left[g^{\prime}\right],\left(g^{\prime} \in M, g^{\prime} \neq g\right)$. Let the elements of $M$ be labelled with the members of a well-ordered set $P$, i.e., let

$$
M=\left\{g_{\pi}: \pi \in P\right\} .
$$

By induction, there exists an orthonormal set $\left\{u_{\pi}: \pi \in P\right\}$ such that for every $\pi^{\prime} \in P$

$$
\left\{\left[g_{\pi}\right]: \pi<\pi^{\prime}\right\}=\left\{\left[u_{\pi}\right]: \pi<\pi^{\prime}\right\} .
$$

The set $\left\{u_{\pi}: \pi \in P\right\}$ must be countable which establishes the lemma.

LEMMA 2.5. Every nonzero ideal I of a regular algebra $A$ contains a minimal idempotent.

Proof. If $I$ contains no minimal idempotent $e_{i}$; then $e_{i} x$ must be zero for every minimal idempotent $e_{i}$ and every $x$ in $I$. It follows that $I$ is the zero ideal, a contradiction.

LEMMA 2.6. Every finite-dimensional ideal I of a regular algebra A is complemented, i.e., there exists a closed ideal $J$ such that $A$ is the direct sum $I+J$.

Proof. Observe that every minimal idempotent $e$ of $A$ either belongs to $I$ or else $e x$ is zero for every $x$ of $I$. Since the set $E$ of minimal orthogonal idempotents of $A$ is finitely linearly independent; only a finite set, say $\left\{e_{i}\right\},(i=1, \ldots, n)$, of minimal idempotents is contained in $I$. Denote by $E_{n}$ the idempotent $e_{1}+\cdots+e_{n}$ of $I$. Every $x$ in $A$ can be written

$$
x=E_{n} x+\left(x-E_{n} x\right) .
$$

If $y$ denotes $x-E_{n} x$, then $e_{i} y$ is zero for every $e_{i}$ contained in $I$. It follows that when $x$ belongs to $I, e_{i} y$ vanishes for every $e_{i}$ in $A$, implying that $y$ is zero. Hence every $x$ of $I$ can be written

$$
x=E_{n} x=\left(x, g_{1}\right) e_{1}+\cdots+\left(x, g_{n}\right) e_{n} .
$$


Thus $I$ is the linear hull $\left(e_{i}\right),\left(e_{i} \in I\right)$. The set of all $y$ such that $e_{i} y$ is zero for $e_{i}$ in $I$ is a closed ideal $J$ containing all elements of the form $x-E_{n} x$ where $x$ is any element of $A$. It follows that $A$ is the direct sum $I+J$.

In the case of $H^{*}$-algebras, orthogonality enables one to extend this lemma to any proper closed ideal of $A$. We recall that the socle of any commutative algebra $A$, Banach or not, is the algebraic sum of the minimal ideals of $A$.

LEMMA 2.7. The socle of a regular algebra $A$ is dense if and only if every proper closed ideal $I$ of $A$ is contained in a maximal regular ideal $R$ of $A$.

Proof. Suppose the socle $\left(e_{i}\right),(i=1,2, \ldots)$, is dense in $A$. If $I$ is a proper closed ideal, then there exists a minimal idempotent $e_{i}$ not contained in $I$. Consequently, $e_{i} I$ is the zero ideal and $I$ is contained in $R_{i}$. Let every proper ideal $I$ of $A$ be contained in a maximal regular ideal $R$ of $A$. Then since $\left[e_{i}\right],(i=1,2, \ldots)$, is a closed ideal not contained in any maximal regular ideal $R$, it must coincide with $A$.

The structural analysis of $H^{*}$-algebras proceeds via the route of orthogonal, complete reducibility. At each stage, one is presented with a direct sum decomposition $I+J$ where $x \in I, y \in J$ imply that $(x, y)$ is zero. Any Hilbert algebra $A$ is said to be well separated if, whenever $A$ is the direct sum $I+J$ of closed ideals $I$ and $J$, there exists a constant $k$, with $0<k<1$, such that $x \in I, y \in J$ imply

$$
|(x, y)| \leqq k\|x\|\|y\| \text {. }
$$

THEOREM 2.8. Let $A$ be a well-separated, regular algebra in which every proper closed ideal is contained in a maximal regular ideal. Then there exists a basis of minimal idempotents, $\left\{e_{i}\right\}$, of $A$ and two sequences of positive numbers, $\left\{d_{i}\right\}$ and $\left\{D_{i}\right\}$, $(i=1,2, \ldots)$, such that

$$
\begin{aligned}
d_{1}\left|x_{1}\right|^{2}+\cdots+d_{n}\left|x_{n}\right|^{2}+\cdots & \leqq\left\|x_{1} e_{1}+\cdots+x_{n} e_{n}+\cdots\right\|^{2} \\
& \leqq D_{1}\left|x_{1}\right|^{2}+\cdots+D_{n}\left|x_{n}\right|^{2}+\cdots
\end{aligned}
$$

for every element $x_{1} e_{1}+\cdots+x_{n} e_{n}+\cdots$ of $A$. Furthermore, there exists a continuous homomorphism of an $H^{*}$-algebra $H_{1}$ into a dense subset of $A$ and a continuous homomorphism of $\mathrm{A}$ into an $\mathrm{H}^{*}$-algebra $\mathrm{H}_{2}$.

Proof. Denote by $E$ the set of all minimal idempotents of $A$. Let $\left\{u_{i}\right\}$ be an orthonormal set of $A$ such that

$$
\left\{\left(e_{i}\right), i=1, \ldots, m\right\}=\left\{\left(u_{i}\right), i=1, \ldots, m\right\}
$$

for every $m$. Since the socle is dense, it follows that the set $\left\{u_{i}\right\}$ is an orthonormal basis of $A$.

For each positive integer $n$, let $I_{n}$ and $E_{n}$ denote the ideal $\left(e_{i}\right),(i=1, \ldots, n)$, and the idempotent $e_{1}+\cdots+e_{n}$ respectively. According to Lemma 2.6, $A$ is a direct sum $I_{n}+J_{n}$ of closed ideals. Consequently, any $z \in A$ can be written $x+y$ with $x \in I_{n}, y \in J_{n}$. It follows that

$$
\|z\|^{2} \geqq(1-k)\left(\|x\|^{2}+\|y\|^{2}\right) .
$$


Also

Finally,

$$
\|z\|^{2} \leqq(1+k)\left(\|x\|^{2}+\|y\|^{2}\right)
$$

$$
a\left(\|x\|^{2}+\|y\|^{2}\right) \leqq\|z\|^{2} \leqq b\left(\|x\|^{2}+\|y\|^{2}\right)
$$

where $0<1-k=a<1<1+k=b$. If $c^{2}(1-k)$ is one, then

$$
\left\|E_{n} z\right\| \leqq c\|z\| \text {. }
$$

Thus the projections of the set $\left\{E_{i}\right\},(i=1,2, \ldots)$, are uniformly bounded by the number $c$.

Now, given any $x$ in $A$ and any positive number $\varepsilon$, there exists an integer $N$ such that if

$$
x_{n}=\left(x, u_{1}\right) u_{1}+\cdots+\left(x, u_{n}\right) u_{n}
$$

then

$$
\left\|x-x_{n}\right\|<\varepsilon /(1+c)
$$

for $n$ greater than $N$. Note that equation (2.09) implies that $x_{n} \in I_{r}$ whenever $r$ exceeds $n$. Now for $N<n<r$, we have

$$
\begin{aligned}
\left\|x-E_{r} x\right\| & \leqq\left\|x-x_{n}\right\|+\left\|x_{n}-E_{r} x\right\| \\
& \leqq\left\|x-x_{n}\right\|+\left\|E_{r}\left(x-x_{n}\right)\right\| \\
& \leqq\left\|x-x_{n}\right\|+c\left\|x-x_{n}\right\| \\
& <\varepsilon .
\end{aligned}
$$

Thus the sequence $\left\{E_{j} x\right\},(j=1,2, \ldots)$, where

$$
E_{j} x=\left(x, g_{1}\right) e_{1}+\cdots+\left(x, g_{j}\right) e_{j}
$$

converges to $x$ for every $x \in A$. Consequently, the set $E$ of minimal idempotents is a basis of $A$.

Repeated applications of inequality (2.10) lead to the following inequality,

$$
\begin{aligned}
\left|x_{1}\right|^{2} a\left\|e_{1}\right\|^{2}+\cdots+\left|x_{n}\right|^{2} a^{n}\left\|e_{n}\right\|^{2} & \leqq\left\|x_{1} e_{1}+\cdots+x_{n} e_{n}\right\|^{2} \\
& \leqq\left|x_{1}\right|^{2} b\left\|e_{1}\right\|^{2}+\cdots+\left|x_{n}\right|^{2} b^{n}\left\|e_{n}\right\|^{2}
\end{aligned}
$$

Define two new sequences, $\left\{d_{i}\right\}$ and $\left\{D_{i}\right\}$, by means of

$$
d_{i}=a^{i}\left\|e_{i}\right\|^{2}, \quad D_{i}=b^{i}\left\|e_{i}\right\|^{2}, \quad(i=1,2, \ldots)
$$

Equation (2.14) asserts that given a sequence $\left\{x_{n}\right\}$ such that

$$
D_{1}\left|x_{1}\right|^{2}+\cdots+D_{n}\left|x_{n}\right|^{2}+\cdots
$$

converges, then there exists an $x$ in $A$ given by

$$
x=x_{1} e_{1}+\cdots+x_{n} e_{n}+\cdots .
$$


Furthermore, it states that if some $x$ in $A$ is given by equation (2.17), then the series

$$
d_{1}\left|x_{1}\right|^{2}+\cdots+d_{n}\left|x_{n}\right|^{2}+\cdots
$$

converges.

These results are quite similar to the standard ones for semisimple, commutative $H^{*}$-algebras. The $H^{*}$-algebras are distinguished by the fact that the two sequences $\left\{d_{i}\right\}$ and $\left\{D_{i}\right\}$ coincide, due to the orthogonality conditions on the minimal idempotents. We are now able to define a continuous homomorphism of an $H^{*}$-algebra $H_{1}$ into a dense subset of $A$ and a continuous homomorphism of $A$ into an $H^{*}$-algebra $H_{2}$.

Let $H_{1}$ denote an $H^{*}$-algebra with a sequence of minimal selfadjoint idempotents $\left\{s_{i}\right\}$ satisfying the conditions that

If

$$
\left\|s_{i}\right\|^{2}=D_{i}
$$

$$
x=x_{1} s_{1}+\cdots+x_{n} s_{n}+\cdots
$$

denotes any element of $H_{1}$, then

$$
\|x\|^{2}=\left|x_{1}\right|^{2} D_{1}+\cdots+\left|x_{n}\right|^{2} D_{n}+\cdots .
$$

The multiplication in $H_{1}$ satisfies a condition of the form

$$
\|x y\| \leqq K^{\prime}\|x\|\|y\|
$$

for every $x, y \in H_{1}$. The mapping $T$ which makes the element $x$ of equation (2.19) correspond to

$$
T(x)=x_{1} e_{1}+\cdots+x_{n} e_{n}+\cdots
$$

is a continuous homomorphism of $H_{1}$ into $A$. Since the $\operatorname{Im} T$ includes all elements of the form, $x_{1} e_{1}+\cdots+x_{n} e_{n}$, the $\operatorname{Im} T$ is dense in $A$. The construction of $H_{2}$ should be clear.

An equation such as equation (2.20) implies that $1 / K^{\prime} \leqq\|e\|$ for an idempotent $e$ of an algebra $A$. However, there is no result in the theory of $H^{*}$-algebras bounding the norms of the minimal selfadjoint idempotents of the algebra. Nevertheless, an $H^{*}$-algebra is somewhat pathological when there is no uniform bound for these. Call a sequence $\left\{x_{n}\right\}$ admissible with respect to the basis $\left\{v_{i}\right\}$ if $x_{1} v_{1}+\cdots+x_{n} v_{n}+\cdots$ converges. For $H^{*}$-algebras with bases of uniformly bounded minimal idempotents $\left\{v_{i}\right\}$, the sequence $\left\{x_{n}\right\}$ and $\left\{x_{p(n)}\right\}$ are either both admissible or both inadmissible, for any permutation $p$ of the integers. An algebra $A$ with a basis of minimal idempotents $\left\{e_{i}\right\}$ is homogeneous if it enjoys this property. I. M. Gel'fand [4] has proved that a homogeneous basis is a Fischer-Riesz basis. Thus we have established most of the following theorem. The remainder of the proof is given in $\$ 4$.

THEOREM 2.9. A well-separated, regular algebra $A$ in which every proper closed 
ideal is contained in a maximal modular ideal is an isomorphic image of a homogeneous $H^{*}$-algebra if and only if $A$ is homogeneous.

We close this section with a characterization of regular algebras in terms of their structure space. We recall that for a commutative Banach algebra the structure space is the set of all maximal modular ideals $M$. Recall that the hull $h(I)$ of an ideal $I$ is the set of all maximal modular ideals containing $I$. The kernel $k(S)$ of a set $S$ of maximal modular ideals is their intersection, a closed ideal. The set $M$ of maximal modular ideals is topologized by defining the closure $\bar{S}$ of a set $S$ of $M$ to be the hull of the kernel of $S$.

THEOREM 2.10. An algebra $A$ is regular if and only if the structure space $M$ of $A$ is discrete in the hull-kernel topology.

Proof. Let $M$ denote the structure space of a regular algebra $A$. Denote by $S_{i}$ the subset of $M$ consisting of all maximal regular ideals other than $R_{i}$. Let $J_{i}$ be $k\left(S_{i}\right)$, the kernel of $S_{i}$. Since $\left(e_{i}, g_{j}\right)$ is zero for $j$ different from $i$, it follows that $J_{i}$ contains the minimal ideal $A e_{i}$. Thus

$$
J_{i}=A e_{i}+J_{i} \cap R_{i}
$$

where the sum is direct. However, if $x$ is an element of $J_{i} \cap R_{\mathfrak{i}}$, then $x$ belongs to the radical and must be zero. Thus $J_{i}$ coincides with the minimal ideal $A e_{i}$. On the other hand, since $\left(e_{i}, g_{j}\right)$ is zero only for $j$ not equal to $i$, it follows that $h\left(J_{i}\right)$ must be $S_{i}$.

Consequently,

$$
S_{i}=h\left(J_{i}\right)=h\left(k\left(S_{i}\right)\right),
$$

and $S_{i}$ is closed in the hull-kernel topology. Therefore $R_{i}$, the complement of $S_{i}$, must be open. Since $R_{i}$ is maximal, it follows that

$$
R_{i}=h\left(R_{i}\right)=h\left(k\left(R_{i}\right)\right)
$$

so that $R_{i}$ is also closed. The structure space $M$ is discrete.

Suppose, on the other hand, that the structure space of a semisimple, commutative $H$-algebra $A$ is discrete. Using the notation $S_{i}$ as above, we see that $S_{i}$ is closed so that

$$
S_{i}=h\left(k\left(S_{i}\right)\right)
$$

Consequently, there exists a nonzero vector $x_{i}$ in $k\left(S_{i}\right)$ which is not in $R_{i}$. It follows that $\left(x_{i}, g_{i}\right)$ is not zero. For each $i$, define $e_{i}$ to be $x_{i} /\left(x_{i}, g_{i}\right)$. Then

$$
\begin{array}{rlrl}
\left(e_{i}, g_{j}\right) & =1, & & i=j, \\
& =0, & i \neq j .
\end{array}
$$

Thus the sequences $\left\{e_{i}\right\},\left\{g_{i}\right\},(i=1,2, \ldots)$, form a biorthogonal system. We note that $\left(e_{i}^{2}-e_{i}, g_{j}\right)$ is zero for every $j$ which implies that

$$
e_{i}^{2}-e=0, \quad \text { or } \quad e_{i}^{2}=e_{i} \text {. }
$$


Consequently, the sequence $\left\{e_{i}\right\}$ consists entirely of idempotents. Furthermore, note that for every $x$

$$
\left(e_{i} x, g_{j}\right)=\left(e_{i}, g_{j}\right)\left(x, g_{j}\right)
$$

This equation implies that $e_{i} x$ is an element of $R_{j}$ whenever $i$ differs from $j$. To the contrary, $e_{i} x$ belongs to $R_{i}$ only when it is the zero vector. In addition, for any $x$ in $H$,

$$
\left(x-e_{i} x, g_{i}\right)=0 .
$$

Hence $A$ is the direct sum $R_{i}+A e_{i}$, i.e., every maximal modular ideal $R_{i}$ is complemented and $A$ is regular.

3. Adjoint algebras. Let $H$ be a Hilbert space on which there exist two binary operations $f$ and $f^{*}$. The standard product of $x, y \in H$ is the image $f(x, y)$ and is denoted by $x y$. The adjoint product is the image $f^{*}(x, y)$ denoted by $x \cdot y$ to distinguish it from the standard product. Assume further that constants $K$ and $K^{*}$ exist such that

$$
\|x y\| \leqq K\|x\|\|y\|, \quad\|x \cdot y\| \leqq K^{*}\|x\|\|y\| .
$$

In addition, there exists a bijection $\pi$ on $H$ with $\pi(x)$ denoted by $x^{*}$ such that $\left\|x^{*}\right\| \leqq b\|x\|,\|x\| \leqq b^{*}\left\|x^{*}\right\|$ for all $x$. The set $H$ is said to form an adjoint algebra $A$ if it is a Hilbert algebra under each of the binary operations and the following equation is satisfied for all $x, y, z \in H$

$$
(x y, z)=\left(y, x^{*} \cdot z\right) .
$$

We limit our considerations to the case where $H$ is a semisimple, commutative $\mathrm{H}$-algebra under each of the binary operations. We do not repeat these assumptions in the following paragraphs. We use the adjectives, standard and adjoint, in the expected way. A standard ideal $I$ of $A$ is a vector subspace which is closed under standard multiplication by any element in $A$. An adjoint maximal modular ideal is a maximal modular ideal under the adjoint product. Given that each of the products is semisimple, the following rules are valid

$$
\begin{aligned}
\left(\varepsilon_{1} x+\varepsilon_{2} y\right)^{*} & =\bar{\varepsilon}_{1} x^{*}+\bar{\varepsilon}_{2} y^{*}, \\
(x y)^{*} & =y^{*} \cdot x^{*}=x^{*} \cdot y^{*}
\end{aligned}
$$

where $x, y$ belong to $H ; \varepsilon_{1}, \varepsilon_{2}$ are complex numbers.

The following lemma is clear.

LEMMA 3.1. The orthogonal complement of a standard (adjoint) ideal I of the adjoint algebra $A$ is an adjoint (standard) ideal $J$ of $A$.

LemMA 3.2. An adjoint algebra $A$ is regular under each of its two products.

Proof. Let $R$ be a standard maximal modular ideal of $A$. Then $A$ is the orthogonal direct sum $R+J$ where $J$ is a one-dimensional adjoint ideal of $A$. It follows 
that $J$ is minimal and contains an idempotent generator $e^{*}$ such that $J$ and $A \cdot e^{*}$ coincide. Let $e$ denote $\pi^{-1}\left(e^{*}\right)$ so that $e$ is a standard idempotent by equation (3.03). For $y \in R, x \in A$,

$$
(y e, x)=\left(y, x \cdot e^{*}\right)=0
$$

so that $y e$ is zero for every element $y \in R$. On the other hand, if $y e$ is zero then $\left(y, x \cdot e^{*}\right)=(y e, x)=0$ for every $x$ which implies that $y \in R$, i.e., $R$ is the annihilator of $e$. Finally, $x=(x-x e)+x e$ for every $x$ in $A$, that is

$$
A=R+A e
$$

is a direct sum decomposition of $A$. Consequently, the standard maximal modular ideal $R$ is complemented and $A$ is regular with respect to the standard product. A similar argument is valid for the adjoint product.

Let $\left\{e_{i}\right\}(i=1,2, \ldots)$ denote the complete set of standard minimal idempotents of the adjoint algebra $A$. Then the set $\left\{e_{i}^{*}\right\}(i=1,2, \ldots)$ where $e_{i}^{*}=\pi\left(e_{i}\right)$, is a complete set of minimal adjoint idempotents of $A$. Let $\left\{g_{i}\right\},(i=1,2, \ldots)$, be the set of associated functionals for the set $\left\{e_{i}^{*}\right\}$. Note that

$$
\delta_{j}^{i}=\left(e_{i}^{*}, g_{i}\right)=\left(e_{i}^{*}, e_{i} g_{i}\right)=\left(g_{i}^{*}, e_{i}\right)
$$

which implies that the set $\left\{g_{i}^{*}\right\}$ where $g_{i}^{*}=\pi\left(g_{i}\right)$ is the set of associated functions for the set $\left\{e_{i}\right\}$. In this section, we use the symbols $\left\{e_{i}\right\},\left\{g_{i}^{*}\right\}$ and $\left\{e_{i}^{*}\right\},\left\{g_{i}\right\},(i=1,2, \ldots)$, to denote the biorthogonal sequences of minimal idempotents and associated linear functions in the cases of the standard and adjoint operations, respectively, of the adjoint algebra $A$.

LEMma 3.3. The standard (adjoint) socle is dense in the adjoint algebra $A$.

Proof. The closed linear hull $\left[e_{i}\right],\left(e_{i} \in A\right)$, is a standard ideal $I$ of $A$. If the standard socle is not dense, then the orthogonal complement $J$ of $I$ is a proper adjoint ideal of $A$ and

$$
A=I+J
$$

Let $y \in A, x \in J$, and $e_{i}^{*}$ be any minimal adjoint idempotent. Then

$$
\left(y, e_{i}^{*} \cdot x\right)=\left(e_{i} y, x\right)=0
$$

so that $e_{i}^{*} \cdot x$ is zero for every adjoint minimal idempotent which implies that $x$ is in the adjoint radical. Thus $J$ is a nonzero ideal contained in the adjoint radical, a contradiction. We do not argue the adjoint case since it is essentially the same.

Lemma 3.4. Let $A$ be an adjoint algebra such that $\pi$ is an involution. Then

$$
(x, y)=\left(y^{*}, x^{*}\right)
$$

for all $x, y \in A$. 
Proof. First, consider the case where one of the elements is a minimal idempotent $e_{i}$. Then for any $x \in A$

$$
\begin{aligned}
\left(e_{i}, x\right) & =\left(e_{i}^{2}, x\right)=\left(e_{i}, e_{i}^{*} \cdot x\right) \\
& =\left(e_{i} x^{*}, e_{i}^{*}\right)=\left(x^{*}, e_{i}^{*} \cdot e_{i}^{*}\right)=\left(x^{*}, e_{i}^{*}\right)
\end{aligned}
$$

Now let

$$
z_{n}=z_{n 1} e_{1}+\cdots+z_{n n} e_{n}
$$

and $y$ be any element of $A$. Then

$$
\begin{aligned}
\left(z_{n}, y\right) & =\left(z_{n 1} e_{1}+\cdots+z_{n n} e_{n}, y\right) \\
& =z_{n 1}\left(e_{1}, y\right)+\cdots+z_{n n}\left(e_{n}, y\right) \\
& =z_{n 1}\left(y^{*}, e_{1}^{*}\right)+\cdots+z_{n n}\left(y^{*}, e_{n}^{*}\right) \\
& =\left(y^{*}, \bar{z}_{n 1} e_{1}^{*}+\cdots+\bar{z}_{n n} e_{n}^{*}\right) \\
& =\left(y^{*}, z_{n}^{*}\right) .
\end{aligned}
$$

We see, in particular, for elements $z_{n}$, that

$$
\left\|z_{n}\right\|^{2}=\left(z_{n}, z_{n}\right)=\left(z_{n}^{*}, z_{n}^{*}\right)=\left\|z_{n}^{*}\right\|^{2} .
$$

Since the socle is dense, the stated result follows via continuity of the mapping $\pi$ and of the inner product.

Lemma 3.5. Let $e_{i}$ be a minimal idempotent of the adjoint algebra $A$. Then

$$
e_{i} e_{i}^{*}=\varepsilon_{i} e_{i} \quad \text { and } \quad e_{i}=\pi_{i} g_{i}
$$

where $\varepsilon_{i}$ and $\pi_{i}$ are complex numbers different from zero.

Proof. First, observe that

$$
\left(e_{i}, e_{j}^{*}\right)=\left(e_{i} e_{i}, e_{j}^{*}\right)=\left(e_{i}, e_{i}^{*} \cdot e_{j}^{*}\right)
$$

which is zero whenever $i$ differs from $j$. However, if $\left(e_{i}, e_{i}^{*}\right)$ is also zero, then $e_{i}$ itself must be zero, an impossibility. It follows that $e_{i}$ must be a nonzero multiple of $g_{i}$, that is

$$
e_{i}=\pi_{i} g_{i}
$$

Furthermore, if $e_{i} e_{i}^{*}$ is zero, then

$$
0=\left(e_{i} e_{i}^{*}, e_{i}^{*}\right)=\left(e_{i}^{*}, e_{i}^{*} \cdot e_{i}^{*}\right)=\left(e_{i}^{*}, e_{i}^{*}\right)
$$

so that $e_{i}^{*}$ is zero, contradicting its definition as an adjoint minimal idempotent. Thus

$$
e_{i} e_{i}^{*}=\varepsilon_{i} e_{i}
$$

where $\varepsilon_{i}$ is not zero.

We wish to observe that in the case of an $H^{*}$-algebra, the minimal idempotent $e_{i}$ is selfadjoint and equation (3.10) holds with $\varepsilon_{i}$ equal to one. An adjoint algebra $A$ is called normal if each $\varepsilon_{i}$ satisfying equation (3.10) is positive. 
THEOREM 3.6. Let $A$ be a normal adjoint algebra in which the minimal idempotents are bounded in norm by $B$. Then $A$ has a basis $\left\{e_{i}\right\}(i=1,2, \ldots)$ of minimal idempotents which is a Fischer-Riesz system.

Proof. It follows from equations (3.09) and (3.10) that

$$
\begin{aligned}
\varepsilon_{i} & =\left(\varepsilon_{i} e_{i}, g_{i}^{*}\right)=\left(e_{i} e_{i}^{*}, g_{i}^{*}\right) \\
& =\left(e_{i}^{*}, g_{i}^{*}\right)=\bar{\pi}_{i}\left(g_{i}^{*}, g_{i}^{*}\right)
\end{aligned}
$$

which proves that $\pi_{i}$ is real and positive.

The following inequalities are valid in $A$ :

$$
\begin{aligned}
1 / B & \leqq\left\|g_{i}\right\| \leqq K, \\
1 / B & \leqq\left\|g_{i}^{*}\right\| \leqq K^{*}, \\
1 / K & \leqq\left\|e_{i}\right\| \leqq B, \\
1 / K^{*} & \leqq\left\|e_{i}^{*}\right\| \leqq B, \\
1 / K^{2} & \leqq \pi_{i} \leqq B^{2} .
\end{aligned}
$$

Now let $x$ be any element in $A$ and denote $\left(x, g_{i}^{*}\right)$ by $x_{i}$. Since the standard socle is dense, there exist sequences

$$
\begin{aligned}
z_{n} & =x_{n 1} e_{1}+\cdots+x_{n n} e_{n}, \\
z_{n}^{*} & =\bar{x}_{n 1} e_{1}^{*}+\cdots+\bar{x}_{n n} e_{n}^{*} \\
& =\pi_{1} \bar{x}_{n 1} g_{1}^{*}+\cdots+\pi_{n} \bar{x}_{n n} g_{n}^{*},
\end{aligned}
$$

such that $x$ is the limit of $z_{n}$. It follows from the continuity of the norm and inner product that

$$
\lim \left\|z_{n}\right\|=\|x\|, \text { and } \lim x_{n j}=x_{j} .
$$

From relations (3.11) and (3.12), we see that

$$
\begin{aligned}
(1 / K)^{2}\left(\left|x_{n 1}\right|^{2}+\cdots+\left|x_{n n}\right|^{2}\right) & \leqq \pi_{1}\left|x_{n 1}\right|^{2}+\cdots+\pi_{n}\left|x_{n n}\right|^{2} \\
& =\left(z_{n}, z_{n}^{*}\right) \leqq\left\|z_{n}\right\|\left\|z_{n}^{*}\right\| \leqq b\left\|z_{n}\right\|^{2} .
\end{aligned}
$$

Given $\varepsilon>0$, there exists an integer $N$ such that

$$
\left\|z_{n}\right\|^{2} \leqq\left(\|x\|^{2}+\varepsilon\right)^{2}
$$

whenever $n>N$. Thus, for this case,

$$
(1 / K)^{2}\left(\left|x_{n 1}\right|^{2}+\cdots+\left|x_{n n}\right|^{2}\right) \leqq b(\|x\|+\varepsilon)^{2},
$$

which implies that, for $v=K^{2} b$,

$$
\left(\left|x_{1}\right|^{2}+\cdots+\left|x_{n}\right|^{2}\right) \leqq v(\|x\|+\varepsilon)^{2}
$$

for every $\varepsilon$. Consequently, we have

$$
\begin{aligned}
\left(\left|x_{1}\right|^{2}+\cdots+\left|x_{n}\right|^{2}\right) & \leqq v\|x\|^{2}, \\
\left(\left|x_{1}\right|^{2}+\cdots+\left|x_{n}\right|^{2}+\cdots\right) & \leqq v\|x\|^{2} .
\end{aligned}
$$


Equation (3.14) implies that the set $\left\{e_{i}\right\}$ of minimal idempotents is a Bessel system. It follows from the results of Bary, that the system $\left\{g_{i}^{*}\right\}$, biorthogonal to it, is a Hilbert system. Therefore, given any sequence $\left\{c_{i}\right\}$ in $l_{2}$, the sequence $\left\{z_{n}\right\}$ given by

$$
z_{n}=c_{1} g_{1}^{*}+\cdots+c_{n} g_{n}^{*}
$$

converges to an element $x$ of $A$. Furthermore, there exists a constant $m$ such that

$$
\|x\| \leqq m\left(\left|c_{1}\right|^{2}+\cdots+\left|c_{n}\right|^{2}+\cdots\right) .
$$

Note that the sequence $\left\{c_{i}\right\}$ belongs to $l_{2}$ if and only if the sequence $\left\{c_{i} \pi_{i}\right\}$ belongs to $l_{2}$. Consequently, if $\left\{c_{i}\right\}$ is in $l_{2}$, then the sequence $\left\{z_{n}\right\}$ where

$$
\begin{aligned}
z_{n} & =c_{1} e_{1}^{*}+\cdots+c_{n} e_{n}^{*} \\
& =c_{1} \pi_{1} g_{1}^{*}+\cdots+c_{n} \pi_{n} g_{n}^{*}
\end{aligned}
$$

converges to an element $x$ in $A$. In addition,

$$
\begin{aligned}
\|x\| & \leqq m\left(\left|\pi_{1} c_{1}\right|^{2}+\cdots+\left|\pi_{n} c_{n}\right|^{2}+\cdots\right) \\
& \leqq m B^{4}\left(\left|c_{1}\right|^{2}+\cdots+\left|c_{n}\right|^{2}+\cdots\right)
\end{aligned}
$$

Thus, we see that the system $\left\{e_{i}^{*}\right\}$ is a Hilbert system. However, $\left\{e_{i}^{*}\right\}$ is a Bessel system by the same sort of argument that proved that $\left\{e_{i}\right\}$ is a Bessel system. It follows that $\left\{e_{i}^{*}\right\}$ and, consequently, $\left\{e_{i}\right\}$ are Fischer-Riesz systems, as was to be shown.

We turn to one further variation in the axioms in which the results follow almost immediately.

Let $A$ be a commutative, semisimple $H$-algebra with an involution $\pi$ where the image $\pi(x)$ is denoted by $x^{*}$. Suppose that $f$ and $f^{*}$ are two inner products on $A$ with $f(x, y)$ and $f^{*}(x, y)$ denoted by $(x, y)$ and $[x, y]$ respectively. In addition, assume there exist positive constants $B$ and $B^{*}$ such that

$$
|(x, y)|^{2} \leqq B^{2}[x, x][y, y], \text { and }|[x, y]|^{2} \leqq\left(B^{*}\right)^{2}(x, x)(y, y) .
$$

The algebra $A$ is called a dual adjoint algebra if the involution and the inner products satisfy the relations

$$
(x y, z)=\left[x, z y^{*}\right]=\left[y, x^{*} z\right] .
$$

TheOREM 3.7. The dual adjoint algebra $A$ has a basis $\left\{e_{i}\right\},(i=1,2, \ldots)$, of minimal idempotents. Furthermore, the basis $\left\{e_{i}\right\}$ is a Fischer-Riesz system if and only if the minimal idempotents are uniformly bounded.

Proof. We define a new inner product on $A$ under which it becomes an $H^{*}$-algebra. For $x, y \in A$, let

$$
\{x, y\}=(x, y)+[x, y] .
$$


Denote the norm of $x$ in this new inner product by $\|x\|$. . It is easy to see that

$$
\begin{aligned}
& |\{x, y\}| \leqq\left(1+B^{*}\right)\|x\|\|y\|, \text { and } \\
& |(x, y)| \leqq\|x\|\|y\| \leqq\|x\|\|\| y \|
\end{aligned}
$$

where $\|x\|^{2}$ denotes $(x, x)$. Furthermore, if $\|x y\| \leqq K\|x\|\|y\|$, then $\|x y\|$ $\leqq K^{\prime}\|x\|\|\| y \|$ where $K^{\prime}$ denotes $K\left(1+B^{*}\right)^{1 / 2}$. Finally,

$$
\{x y, z\}=\left\{y, x^{*} z\right\}=\left\{x, z y^{*}\right\}
$$

so that $A$ is a semisimple, commutative $H^{*}$-algebra in this new norm. We denote the "two" normed algebras by $A(, \quad)$ and $A\{$,$\} respectively.$

The fundamental theorem on $H^{*}$-algebras asserts that $A\{$,$\} has an ortho-$ normal basis $\left\{e_{i}\right\},(i=1,2, \ldots)$, of minimal selfadjoint idempotents. The identity mapping of $A\{$,$\} onto A(, \quad)$ is a continuous automorphism. It follows that the set $\left\{e_{i}\right\}$ is a basis of minimal idempotents in the algebra $A($,$) , the original$ dual adjoint algebra. It is easy to see that $\left\{e_{i}\right\}$ is a Fischer-Riesz basis in each algebra if and only if the minimal idempotents are uniformly bounded.

4. Examples. The following examples were suggested by the results of $\mathrm{N}$. Bary [3] in which the most natural generalization of orthonormal base is developed. Her more important results for biorthogonal systems $\left\{e_{i}\right\},\left\{g_{i}\right\},(i=1,2, \ldots)$, in the separable Hilbert space $H$ are in terms of three basic concepts. The complete system $\left\{e_{i}\right\}$ is said to be a Bessel system if given any $x \in H$, the coefficients $\left\{\left(x, g_{i}\right)\right\}$ belong to $l_{2}$. The complete system $\left\{g_{i}\right\}$ is said to be a Hilbert system if given any sequence $\left\{c_{i}\right\}$ in $l_{2}$ there exists a unique $x \in H$ such that $\left\{c_{i}\right\}$ coincides with the sequence $\left\{\left(x, e_{i}\right)\right\}$. A complete system $\left\{e_{i}\right\}$ is said to be a Fischer-Riesz system if it is both a Bessel and a Hilbert system. The fundamental results of Bary's are: A system $\left\{e_{i}\right\}$ is a Fischer-Riesz system if and only if it is the image of every orthonormal basis $\left\{u_{i}\right\}$ of $H$ under some continuous automorphism, not necessarily isometric, of $H$. A system $\left\{e_{i}\right\}$ is a Bessel system if and only if every orthonormal basis $\left\{u_{i}\right\}$ is the image of $\left\{e_{i}\right\}$ under some continuous endomorphism of $H$. A system $\left\{e_{i}\right\}$ is a Hilbert system if and only if it is the image of every unitary basis $\left\{u_{i}\right\}$ under some continuous endomorphism of $H$.

The results have the following additional interpretations. Let $\left\{e_{i}\right\},\left\{g_{i}\right\}$ form a biorthogonal system. Let $x$ be in $H$ and consider the following two inequalities:

$$
\begin{aligned}
& m\left(\left|\left(x, g_{1}\right)\right|^{2}+\cdots+\left|\left(x, g_{n}\right)\right|^{2}+\cdots\right) \leqq\|x\|^{2} . \\
& \|x\|^{2} \leqq M\left(\left|\left(x, g_{1}\right)\right|^{2}+\cdots+\left|\left(x, g_{n}\right)\right|^{2}+\cdots\right) .
\end{aligned}
$$

A Fischer-Riesz system $\left\{e_{i}\right\}$ is a basis such that constants $m$ and $M$ exist for which both inequalities (4.01) and (4.02) are valid whenever $x$ belongs to $H$. Although a complete Hilbert system $\left\{e_{i}\right\}$ need not be a basis, there exists a constant $M$ such that inequality (4.02) is valid for every $x$ in $H$. Furthermore, a complete Bessel system $\left\{e_{i}\right\}$ need not be a basis, but there exists a constant $m$ such that inequality (4.01) is valid for every $x$ in $H$.

We need the following Lemmas before considering our examples. 
LEMMA 4.1. Let the Hilbert space $H$ be the direct sum $I+J$ of the nonorthogonal, closed subspaces $I$ and $J$. Let $P$ be the projection of $H$ on $I$ along $J$. Then if $u \in I$, $v \in J$

$$
|(u, v)| \leqq k\|u\|\|v\|, \text { and } 0<k<1 .
$$

Proof. Let $u$ and $v$ be elements of $I$ and $J$, respectively, each being of unit norm. Since $u$ and $v$ are linearly independent, $|(u, v)|<\|u\|\|v\|=1$.

If $r$ is the vector $[u-(u, v) v] /\left[1-|(u, v)|^{2}\right]$, then it is easy to see that

$$
\|P\|^{2} \geqq\|r\|^{2}=1 /\left[1-|(u, v)|^{2}\right] \geqq 1 .
$$

From which it follows that

$$
|(u, v)|^{2} \leqq\left\{\|P\|^{2}-1\right\} /\|P\|^{2}<k^{2}<1 .
$$

For general $u$ and $v$, we have the desired result:

$$
|(u, v)| \leqq k\|u\|\|v\| \text {. }
$$

Whenever $T$ is a continuous automorphism of a Hilbert space $H$ onto itself, $T$ has a continuous inverse $T^{-1}$ such that $1 \leqq\|T\|\left\|T^{-1}\right\|$.

LEMMA 4.2. Let $T$ be a continuous automorphism of a Hilbert space $H$ onto itself. Then, if $\|T\|\left\|T^{-1}\right\|$ is one, $T$ is essentially unitary, i.e., there exists a positive number $r$ and $a$ unitary transformation $U$ such that

$$
T=r U \text {. }
$$

Proof. Suppose there exist vectors $x_{1}, x_{2}$ in $H$ such that

Then

$$
\left\|x_{1}\right\|=\left\|x_{2}\right\|=1, \quad\left\|T x_{1}\right\|<\left\|T x_{2}\right\| .
$$

so that

$$
1 /\left(\left\|T^{-1}\right\|\right) \leqq\left\|T x_{1}\right\|<\left\|T x_{2}\right\| \leqq\|T\|
$$

a contradiction. Thus

$$
1<\|T\|\left\|T^{-1}\right\|
$$

$$
\left\|T x_{1}\right\|=\left\|T x_{2}\right\|=r^{2}>0
$$

whenever $x_{1}$ and $x_{2}$ are of norm one. It follows that

$$
((T / r) x,(T / r) x)=(x, x)
$$

for every $x$, i.e., $(T / r)$ is an isometry and, consequently, must be unitary.

Let $\left\{e_{i}\right\},\left\{g_{i}\right\}$ be a biorthogonal system where both $\left\{e_{i}\right\}$ and $\left\{g_{i}\right\}$ are Fischer-Riesz systems. Let $\left\{u_{i}\right\}$ be an orthonormal basis of $H$ and $T$ be the continuous automorphism of $H$ such that

$$
e_{i}=T u_{i}, \quad \text { and } \quad 1 /\left(\left\|T^{-1}\right\|\right) \leqq\left\|e_{i}\right\| \leqq\|T\| .
$$

The continuous automorphism $T^{\prime}=\left(T^{*}\right)^{-1}$ has the property that

$$
g_{i}=T^{\prime} u_{i}, \text { and } 1 /\left\|T^{*}\right\| \leqq\left\|g_{i}\right\| \leqq\left\|T^{\prime}\right\| \text {. }
$$


REMARK 4.3. Now let $R \cup S$ be any partition of the integers $Z$ into disjoint subsets and

$$
\begin{aligned}
& I^{\prime}=\left[u_{i}\right], \quad(i \in R) ; \quad J^{\prime}=\left[u_{i}\right], \quad(i \in S) ; \\
& I=\left[e_{i}\right], \quad(i \in R) ; \quad J=\left[e_{i}\right], \quad(i \in S) .
\end{aligned}
$$

Then $H$ is the orthogonal direct sum $I^{\prime}+J^{\prime}$ of the subspaces $I^{\prime}$ and $J^{\prime}$. It follows from the fact that $T$ is a continuous automorphism that $H$ is also the direct sum $I+J$.

LEMMA 4.4. The subspaces $I$ and $J$ are well separated, i.e., there exists a constant $k$ with $0<k<1$ such that if $u \in I, v \in J$ then $|(u, v)| \leqq k\|u\|\|v\|$ for any choice of the partitioning sets $R$ and $S$.

Proof. Let $P$ and $P^{\prime}$ denote the orthogonal projections on the subspaces $I^{\prime}$ and $J^{\prime}$ respectively. The projections on $I$ and $J$ are given by

$$
E=T(P) T^{-1} \quad \text { and } \quad E^{\prime}=T\left(P^{\prime}\right) T^{-1}
$$

from which it follows that each of them is bounded by the constant $\|T\|\left\|T^{-1}\right\|$. It follows from Lemma 4.1 that if $k$ is any constant such that

$$
\left(\left(\|T\|\left\|T^{-1}\right\|\right)^{2}-1\right) /\left(\|T\|\left\|T^{-1}\right\|\right)^{2}<k^{2}<1
$$

then $u \in I, v \in J$ implies $|(u, v)| \leqq k\|u\|\|v\|$.

EXAMPLE 4.5. Let $\left\{e_{i}\right\}$ be a Fischer-Riesz basis for the separable Hilbert space $H$. If

$$
x=x_{1} e_{1}+\cdots+x_{n} e_{n}+\cdots, \quad y=y_{1} e_{1}+\cdots+y_{n} e_{n}+\cdots
$$

are any two elements in $H$, let the product $x y$ be defined by

$$
x y=x_{1} y_{1} e_{1}+\cdots+x_{n} y_{n} e_{n}+\cdots \text {. }
$$

Then it follows from inequalities (4.01) and (4.02) that

$$
\begin{aligned}
\|x y\| & \leqq M\left(\left|x_{1} y_{1}\right|^{2}+\cdots+\left|x_{n} y_{n}\right|^{2}+\cdots\right) \\
& \leqq M\left(\left|x_{1}\right|^{2}+\cdots+\left|x_{n}\right|^{2}+\cdots\right)\left(\left|y_{1}\right|^{2}+\cdots+\left|y_{n}\right|^{2}+\cdots\right) \\
& =\left(M / m^{2}\right)\left[m\left(\left|x_{1}\right|^{2}+\cdots+\left|x_{n}\right|^{2}+\cdots\right)\right]\left[m\left(\left|x_{1}\right|^{2}+\cdots+\left|x_{n}\right|^{2}+\cdots\right)\right] \\
& \leqq\left(M / m^{2}\right)\|x\|^{2}\|y\|^{2} .
\end{aligned}
$$

Consequently, we see that $H$ becomes a Hilbert algebra $A$ with this definition of product. Denote by $R_{j}$ the set of all linear combinations

$$
x=x_{1} e_{1}+\cdots+x_{n} e_{n}+\cdots
$$

where the coefficient $x_{j}$ is zero. Clearly, $R_{j}$ is a maximal modular ideal. The algebra $A$ is semisimple since the intersection of all the maximal modular ideals in the set $\left\{R_{i}\right\},(i=1,2, \ldots)$, is the zero ideal. The basis $\left\{e_{i}\right\}$ is clearly a maximal set of minimal idempotents. Suppose that $R$ is any maximal modular ideal of $A$. Since $R$ is closed, there exists an $e_{j}$ which is not contained in $R$. From this, it follows that $R$ must be contained in $R_{j}$ and, consequently, must coincide with $R_{j}$. The minimal ideal $A e_{j}$ is complementary to $R$. Thus $A$ is a regular algebra. The sequence $\left\{g_{i}\right\}$ 
biorthogonal to $\left\{e_{i}\right\}$ is the set of multiplicative elements associated with the set $\left\{R_{i}\right\}$ of maximal modular ideals. Given that $A$ is the direct sum $I+J$ of two proper closed ideals, then $I$ coincides with $\left[e_{i}\right],\left(e_{i} \in I\right)$, and $J$ coincides with $\left[e_{i}\right],\left(e_{i} \in J\right)$. It follows from Lemma 4.4 that $A$ is well separated. Inequalities (4.01) and (4.02) imply that $A$ is homogeneous. Thus we see that $A$ is an example of a homogeneous, semisimple, commutative well-separated regular algebra in which every proper closed ideal is contained in a maximal regular ideal.

Furthermore, the mapping $T^{-1}$ of equation (4.04) is an isomorphism of $A$ onto an $H^{*}$-algebra $H$ generated by taking the orthonormal basis $\left\{u_{i}\right\}$ as the selfadjoint minimal idempotents of $H$. Consequently, every $H$-algebra $A$ with a set $\left\{e_{i}\right\}$ of minimal idempotents which form a Fischer-Riesz basis for the space $A$ is isomorphic to an $H^{*}$-algebra. This result shows in particular that a homogeneous, wellseparated, regular algebra $A$ in which every proper closed ideal is contained in a maximal regular ideal is isomorphic to an $H^{*}$-algebra. When the isomorphism is also isometric, it is easy to see as well that the original algebra is actually an $H^{*}$-algebra and the isomorphism is a ${ }^{*}$-isomorphism.

We note that the definition of the multiplication can be framed somewhat differently. If $x, y \in A$, then the product $x y$ can be defined to be that unique element $z$ in $A$ such that

$$
\left(z, g_{i}\right)=\left(x, g_{i}\right)\left(y, g_{i}\right) \text {. }
$$

A second product $x \cdot y$ can be defined to be that unique element $z^{\prime}$ in $A$ such that

$$
\left(z^{\prime}, e_{i}\right)=\left(x, e_{i}\right)\left(y, e_{i}\right)
$$

This last definition is equivalent to defining $x \cdot y$ for

$$
\begin{aligned}
& x=x_{1} g_{1}+\cdots+x_{n} g_{n}+\cdots, \\
& y=y_{1} g_{1}+\cdots+y_{n} g_{n}+\cdots
\end{aligned}
$$

to be given by

$$
x \cdot y=x_{1} y_{1} g_{1}+\cdots+x_{n} y_{n} g_{n}+\cdots .
$$

A sequence $\left\{x_{n}\right\}$ is admissible for the Fischer-Riesz basis $\left\{e_{i}\right\}$ if and only if it is admissible for the system $\left\{g_{i}\right\}$. Consequently, the mapping $\pi$ defined by

$$
\pi\left(x_{1} e_{1}+\cdots+x_{n} e_{n}+\cdots\right)=\bar{x}_{1} g_{1}+\cdots+\bar{x}_{n} g_{n}+\cdots
$$

is a bijection on $A$. Note that $\pi$ is usually not an involution; however, if we denote $\pi(x)$ by $x^{*}$, then

$$
(x y, z)=\left(x, z \cdot y^{*}\right)=\left(y, x^{*} \cdot z\right)
$$

so that $A$ is an example of an adjoint algebra.

We briefly consider an algebra $A$ which is a continuous automorphic image of an $H^{*}$-algebra $H$ whose minimal idempotents $\left\{s_{i}\right\}$ are uniformly bounded by $B$. If $T$ is the given automorphism, then $T$ maps the set of all minimal selfadjoint 
idempotents $\left\{s_{i}\right\}$ of $H$ onto the set of all minimal idempotents $\left\{e_{i}\right\}$ of $A$. It is easy to see that $\left\{e_{i}\right\}$ is a Fischer-Riesz system so that all of the results on algebras with a Fischer-Riesz basis of minimal idempotents apply to $A$. It should be clear now that if $A$ is an algebra with a Fischer-Riesz basis of minimal idempotents, then $A$ is a continuous isomorphic image of any $H^{*}$-algebra $H$ whose minimal idempotents are uniformly bounded. This statement finishes the proof of Theorem 2.9 and completes our discussion of this particular kind of algebra.

We end with a counterexample:

EXAMPLE 4.6. Let $A$ be an $H^{*}$-algebra whose maximal set of selfadjoint minimal idempotents $\left\{s_{i}\right\}$ has the property that $\left\|s_{k}\right\|$ is equal to $k$. It is easy to see that $A$ is a well-separated, regular algebra in which every proper closed ideal is contained in a maximal regular ideal. Nevertheless, it is clear that there exists no continuous automorphism carrying a unitary basis $\left\{u_{i}\right\}$ onto the set of minimal idempotents $\left\{s_{i}\right\}$. Consequently, the set $\left\{s_{i}\right\}$ is not a Fischer-Riesz basis and $A$ is not homogeneous.

\section{REFERENCES}

1. W. Ambrose, Structure theorems for a special class of Banach algebras, Trans. Amer. Math. Soc. 57 (1945), 364-386.

2. - The $L^{2}$-system of a unimodular group, Trans. Amer. Math. Soc. 65 (1949), 27-48.

3. N. K. Bary, Biorthogonal systems and bases in Hilbert space, Učen. Zap. 148 Matematika (1951), 69-107. (Russian)

4. I. M. Gel' fand, A remark on the work of N. K. Bary, "Biorthogonal systems and bases in a Hilbert space," Učen. Zap. 148 Matematika (1951), 224-225. (Russian)

5. E. R. Keown, Thesis, (unpublished), Mass. Inst. of Tech., Cambridge, 1950.

6. —, Reflexive Banach algebras, Proc. Amer. Math. Soc. 6 (1955), 252-259.

7. S. S. Levin, Über einige mit der Konvergenz im Mittel verbundenen Eigenschaften von Funktionenfolgen, Math. Z. 32 (1930), 491-511.

8. N. Nakano, Hilbert algebras, Tôhoku Math. J. (2) 2 (1950), 4-23.

9. M. Rajagopalan, H-algebras, J. Indian Math. Soc. 25 (1961), 1-25.

10. C. L. Rickart, General theory of Banach algebras, Van Nostrand, Princeton, N. J., 1960.

11. P. P. Saworotnow, On a generalization of the notion of $H^{*}$-algebra, Proc. Amer. Math. Soc. 8 (1957), 47-55.

12. - On the imbedding of a right complemented algebra in Ambrose's $H^{*}$-algebra, Proc. Amer. Math. Soc. 8 (1957), 56-62.

13. - On a realization of a complemented algebra, Proc. Amer. Math. Soc. 15 (1964), 964-966.

14. M. F. Smiley, Right $H^{*}$-algebras, Proc. Amer. Math. Soc. 4 (1953), 1-4.

15. J. F. Smith and P. P. Saworotnow, On some classes of scalar-product algebras, Pacific J. Math. 11 (1961), 739-750.

16. O. Takenouchi, On the maximal Hilbert algebras, Tôhoku Math. J. (2) 3 (1951), 123-131.

17. C. W. Conatser, M.A. Thesis, (unpublished), Texas A\&M Univ., College Station, 1963.

TEXAS A\&M UNIVERSITY, College Station, Texas 